\title{
Von sozialen Bewegungen lernen: Ein Impuls für deutsche Gewerkschaften
}

Wie können Gewerkschaften in einem gewandelten, neoliberalen Umfeld erfolgreich agieren? Die Privatisierung der städtischen Kliniken in Hamburg - bei der seitens der Gewerkschaft sowohl der Weg der Kooperation wie der der Konfrontation beschritten wurdegibt ein bemerkenswertes Beispiel für den Wandel von Arbeitgeber- und Gewerkschaftsstrategien. Mit Anleihen bei sozialen Bewegungen können Gewerkschaften, so zeigt die Erfahrung, solche Konflikte nicht nur besser bestehen, sondern gestärkt aus ihnen hervorgehen. ${ }^{1}$

\section{Einleitung}

Der Hamburger Landesbetrieb Krankenhäuser (LBK) wurde 1995 als eine wirtschaftlich und rechtlich eigenständige Anstalt öffentlichen Rechts gegründet, um die Umstrukturierung der Krankenhäuser umzusetzen. Dies geschah in enger Absprache mit den Betriebsräten. Nachdem die Sozialdemokraten im Jahr 2001 die Wahlen in Hamburg verloren hatten, strebte der Hamburger Senat jedoch die Privatisierung der Krankenhäuser an. Für Beschäftigte wie Patienten stand viel auf dem Spiel: Der LBK war, nach Airbus, Hamburgs zweitgrößter Arbeitgeber und verfügte über die Hälfte aller Krankenhausbetten in der Stadt. Auch wenn die Gewerkschaft ver.di und die Betriebsräte eine Privatisierung nicht verhindern konnten, so gab es dank eines bewegungsgewerkschaftlichen Ansatzes (Social Movement Unionism) doch Mobilisierungserfolge. Die Zahl der Gewerkschaftsmitglieder unter den Klinikbeschäftigten verdoppelte sich zudem in der anschließenden Tarifauseinandersetzung. In der Folge wurden zuvor erreichte tarifliche Standards trotz Privatisierung erhalten.

Dieses Beispiel zeigt: Gewerkschaften sind nicht nur in den Arbeitsbeziehungen und auf nationaler Ebene institutionell verankert, sondern sie sind auch mit der Lokalpolitik und der Zivilgesellschaft verflochten. Wenn Regierungsparteien und Arbeitgeber ihr Interesse an Sozialpartnerschaft verlieren, können Gewerkschaften ihre Strategien ändern, mit dem Ergebnis, dass örtliche Gewerkschaftsorganisationen erstarken und ihre Ziele durchsetzen können. Offenbar sind Gewerkschaften auch eher bereit auf Mobilisierung zu setzen und Koalitionen einzugehen, um Umstrukturierungen entgegenzuwirken, wenn ihre traditionellen, institutionell geprägten Einflussmöglichkeiten gegenüber Regierung und den Arbeitgebern schwinden. Das bewegungsgewerkschaftliche Konzept kann dabei auf lokaler Ebene revitalisierend wirken. Allerdings reicht dieser Ansatz allein nicht aus, dem starken äußeren, politischen wie ökonomischen Druck zu begegnen, der auf Tarifverhandlungen in Deutschland einwirkt und sie verändert.

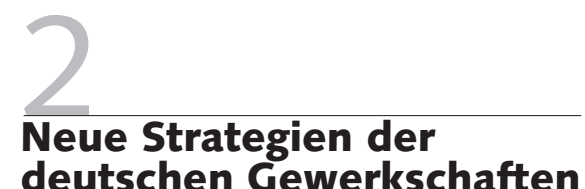

Noch während der 1980er und 1990er Jahre hielten die meisten angelsächsischen Beobachter die deutschen Arbeitsbeziehungen im Vergleich zu denen ihrer Heimatländer für höchst attraktiv (Turner 1991; Katz/Darbishire 2000). Während die industriellen Beziehungen Großbritanniens damals als ein Teufelskreis aus Misstrauen, militantem Widerstand und industriellem Niedergang angesehen wurden, schien Deutschland sich in einer Erfolgsspirale aus hohen Löhnen, hoher Qualifikation, Arbeitsfrieden und wirtschaftlichem Wachstum zu befinden. Während amerikanische Gewerkschaften wechselnden Management-Moden unterworfen und mehr oder weniger dazu verdammt schienen, lediglich als Juniorpartner des Managements aufzutreten, präsentierten sich die deutschen $\mathrm{Ge}$ werkschaften, gestützt auf gesetzliche Regelungen und entsprechende Mobilisierungskapazitäten, als starke Akteure.

Das Modell Deutschland hatte jedoch für die Gewerkschaften auch eine Kehrsei- te, die sich aber erst zeigte, als sie stärker unter Beschuss gerieten. Als in den 1990er Jahren die Tarifbindung abnahm, fanden sich die Gewerkschaften zunehmend darauf beschränkt, sich mit der Schrumpfung ihrer verbliebenen Hochburgen in den Großunternehmen zu befassen und mit den Arbeitgebern über Personalabbau zu verhandeln (Streeck 2001). Co-Management auf Unternehmens- und Branchenebene verstärkte die Ungleichheiten auf dem Arbeitsmarkt (Thelen/Kume 2006), Flächentarifverträge wurden durch betriebliche Bündnisse zur Beschäftigungssicherung in einzelnen Unternehmen unterminiert (Seifert/Massa-Wirth 2005). Die Krise der Gewerkschaften und die damit verbundenen Entwicklungen boten nunmehr Beobachtern aus den USA ein vertrautes Bild.

"Social movement unionism", also bewegungsgewerkschaftliche Ansätze in Anlehnung an Formen und Praxis anderer so-

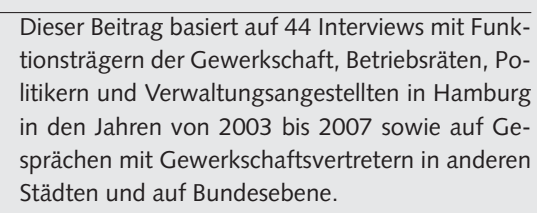
tionsträgern der Gewerkschaft, Betriebsräten, Politikern und Verwaltungsangestellten in Hamburg in den Jahren von 2003 bis 2007 sowie auf Gesprächen mit Gewerkschaftsvertretern in anderen Städten und auf Bundesebene.

Ian Greer, Ph. D., wissenschaftlicher Mitarbeiter am Leeds University Centre for Employment Relations Innovation and Change (CERIC). Arbeitsschwerpunkte: Gewerkschaftsstrategien, Kommunalpolitik sowie verg/eichende industrielle Beziehungen. e-mail: I. C.Greer@leeds.ac.uk

Übersetzung aus dem Englischen: Kristina Vaillant. 
zialer Bewegungen, wurde in den USA als Begriff für eine alternative Gewerkschaftsstrategie geprägt. ${ }^{2}$ Lopez (2004, S. 10f.) definiert den Wandel hin zur Bewegungsgewerkschaft als einen gewerkschaftlichen Veränderungsprozess, der neben breiten gesellschaftlichen Koalitionen und politischen Netzwerken die Mobilisierung und Organisierung der Beschäftigten an der Basis sowie die Einbettung gewerkschaftlicher Forderungen in einen übergreifenden konzeptionellen Rahmen sozialer Gerechtigkeit beinhaltet. Baccaro, Hamann und Turner (2003) argumentieren, die Entstehung von bewegungsgewerkschaftlichen Ansätzen sei in den USA und in Großbritannien durch die kulturellen und institutionellen Rahmenbedingungen begünstigt worden. Angriffe von Arbeitgebern und Regierung auf die Gewerkschaftsbewegung seien dort wesentlich direkter gewesen, sodass die Gewerkschaften stärker unter Druck gestanden hätten, Handlungsalternativen zu entwickeln.

Allerdings zeigen sich in Deutschland inzwischen auch vermehrt Anzeichen einer Gewerkschaftsarbeit, die sich an Vorbildern sozialer Bewegungen orientiert. Studien zur Arbeit von Betriebsräten und Gewerkschaftsfunktionären bei der IG Metall verzeichnen einen Anstieg der Aktivitäten außerhalb des Arbeitsplatzes als Reaktion auf den wirtschaftlichen Wandel in den Regionen und auf Angriffe der Arbeitgeberseite gegen etablierte Strukturen in den Arbeitsbeziehungen (Dörre/Röttger 2006). Im Einzelhandel gibt es ebenfalls Beispiele: Mit der Unterstützung einzelner Politiker und der Kirche konnte die Gewerkschaft Handel, Banken und Versicherungen (HBV) während der 1990er Jahre bei der Drogeriemarktkette Schlecker Fuß fassen und Betriebsratsstrukturen etablieren (Huhn 2001). Seit 2004 versucht ver.di an diese Erfolgsgeschichte beim Lebensmitteldiscounter Lidl anzuknüpfen. Bei innergewerkschaftlichen Diskussionen gewinnt die Frage nach neuen Wegen der Mobilisierung an Bedeutung. Verstärkt wird beispielsweise auf das Know-how von Kampagnenberatern zurückgegriffen, die sich, wie das Beratungsnetzwerk Orka (Organisierung und Kampagnen), teilweise explizit an amerikanischen Erfahrungen orientieren. CoManagement und Dezentralisierung von Tarifpolitik sind somit nicht alternativlos. Wann aber wird auf alternative Strategien zurückgegriffen und wie sind ihre Möglichkeiten und Grenzen einzuschätzen?

\section{Die Umstrukturierung der städtischen Kliniken in Hamburg}

In den Krankenhäusern der Hansestadt Hamburg vollzog sich ein Wandel, wenngleich die Strukturen der Arbeitsbeziehungen formal gesehen stabil blieben. Mit der Privatisierungsentscheidung im Jahr 2001 begann die Sozialpartnerschaft zu bröckeln, obwohl Gewerkschaften, Arbeitgeberorganisationen und Tarifverträge als Institutionen intakt blieben. Weil der Arbeitgeber mit traditionellen Kooperationsformen brach, suchte auch ver.di nach anderen Einflussmöglichkeiten. Obwohl sich bei der gesetzlichen Regelung der Mitbestimmung und der Tarifverhandlungen nichts geändert hat, werden diese Institutionen geschwächt, wenn die Sozialpartnerschaft zerbricht.

\subsection{UMSTRUKTURIERUNG ZU ZEITEN DER SOZIALPARTNERSCHAFT}

Bis 2001 regierte in Hamburg die SPD, zeitweise in Koalition mit FDP oder Grünen. Durch die engen Verbindungen zur SPD hatten die Gewerkschaften einen Zugang zu Senat und Bürgerschaft. Wie in anderen Städten auch waren die Manager in den stadteigenen Betrieben in der Regel Mitglied einer politischen Partei, in Hamburg oft der SPD. Gewerkschaftsvertreter im öffentlichen Dienst verfügten daher neben den offiziellen Mitbestimmungsorganen über weitere Wege der Einflussnahme auf Gesetzgebung und Verwaltung.

In gewisser Weise entsprach die Organisation gewerkschaftlicher Interessenvertretung im Gesundheitssektor, wie im öffentlichen Sektor in Deutschland insgesamt, dem neokorporatistischen Modell, das die vergleichende politische Ökonomie in den 1970er und 1980er Jahre beschrieben hat (Turner 1991). Zentrale Tarifvereinbarungen strukturierten bis 2005 den gesamten öffentlichen Dienst, einschließlich des Gesundheitswesens. 2005 wurde mit dem Tarifvertrag für den öffentlichen Dienst (TVöD) ein neues Tarifwerk vereinbart, dem jedoch die Landesregierungen zunächst nicht beitraten. Seit 2006 hat die einstmals einheitliche Tariflandschaft mit den getrennten Tarifverträgen, die ver.di und der Marburger Bund für die Ärzte abgeschlossen haben sowie mit den schließlich im Ergebnis des Arbeitskampfs 2006 je nach Bundesland unterschiedlichen tariflichen Arbeitszeitregelungen, deutliche Risse bekommen (Bispinck 2006). Die betriebliche Mitbestimmung ist im öffentlichen Dienst durch das Personalvertretungsgesetz geregelt, dass zwar teilweise hinter dem Betriebsverfassungsgesetz zurückbleibt, gleichwohl aber immer noch betriebliche Interessenvertretung auf vergleichsweise hohem Niveau sichert.

Seit Mitte der 1990er Jahre waren die kommunalen Kliniken in Hamburg das Ziel von Modernisierungsbemühungen. Bürgerschaftsabgeordnete fanden, dass die Krankenhäuser nicht effizient genug arbeiteten, um dem erhöhten Kostendruck standzuhalten, der von der Gesundheitspolitik der konservativen Bundesregierung und den Krankenkassen ausging. 1995 beschlossen Senat und LBK-Management Einsparungen in Höhe von 200 Mio. DM. Daraufhin folgte im nächsten Jahr die Schließung des historischen Hafenkrankenhauses in St. Pauli. Letzteres führte zur Besetzung des Gebäudes durch Gewerkschafts- und Stadtteilaktivisten, bis es zu einer umfangreichen Verhandlungslösung kam.

Diese Einigung wurde von Gewerkschaftsvertretern, Personalräten, Managern und Politikern - zumeist Angehörige der SPD - mit dem Ziel ausgehandelt, die Leistungsfähigkeit der Hamburger Krankenhäuser zu steigern und gleichzeitig betriebsbedingte Kündigungen zu vermeiden. Gerade erst war ein neuer Personalrat gewählt, der in der vertrauensvollen $\mathrm{Zu}$ sammenarbeit eine wirksamere Strategie als in der Konfrontation sah. Der Verwaltungsdirektor des LBK, ein SPD-Mitglied, wurde vom Senat damit betraut, eine Betriebsvereinbarung mit den Belegschaftsvertretern auszuhandeln. SPD-Politiker in Bürgerschaft und Senat, inklusive des DGB-Vorsitzenden, taten ihren Teil, indem sie den Gesetzentwurf zur Gründung des neuen LBK formulierten und absegneten. Die Zugehörigkeit aller Beteiligten zur SPD und das gemeinsame Ziel, sowohl die Be-

\footnotetext{
2 Der Begriff hat eine lange Geschichte und wird z. B. im Kontext der Entstehung von Gewerkschaften im öffentlichen Dienst in Kalifornien in den 1970er Jahren (Johnston 1994) oder globaler Solidarität gegen den Neoliberalismus (Moody 1997) unterschiedlich, auch normativ, verwendet.
} 
triebsvereinbarung wie die Gesetzgebung zur Modernisierung der städtischen Kliniken zu nutzen, führten zu einem Kompromiss, der als Paradebeispiel für die deutschlandtypische „sozialverträgliche“ Kostenreduzierung gelten kann. Ein Regelwerk entstand, das die Arbeit in dieser neuen Organisation regulieren sollte. Es beinhaltete auch einen sogenannten Kooperationsvertrag, der die Struktur zusätzlicher Mitbestimmungsausschüsse zur Mitgestaltung der Veränderungsprozesse und ihre Anhörungsrechte festlegte. Die Bürgerschaft verabschiedete ein Gesetz, das eine neue Organisationsstruktur für den LBK festlegte und auch ein Rückkehrrecht für Krankenhausangestellte, die den LBK verlassen wollten, in den verbleibenden öffentlichen Dienst der Stadt festschrieb.

Die Vereinbarungen ermöglichten dem LBK Einsparungen auf verschiedenen Ebenen. Zum einen gliederte das Management die Reinigungsdienste, Apotheken, Wäschereien, den Einkauf, die Logistik, das Gebäudemanagement und die Ausbildung in $100 \%$ ige Tochterfirmen bzw. neu geschaffene Dienstleistungsbetriebe aus. Auch wenn für diese ausgegliederten Bereiche zum größten Teil noch die Vereinbarungen des öffentlichen Dienstes galten, so schuf diese Umstrukturierung doch Raum für den Abbau von Personal. Zum anderen akzeptierte die ÖTV die Schließung des Hafenkrankenhauses, den Abbau von 3000 der insgesamt 15.000 Stellen im Unternehmen sowie verschiedene Maßnahmen zur Vermeidung betriebsbedingter Kündigungen. Die Vereinbarung erlaubte es dem Management, mit Hilfe genauerer Messmethoden einen sogenannten „Normalisierungsprozess" innerhalb der Kliniken mit dem Ziel einzuleiten, Personal abzubauen und die Kosten um $15 \%$ zu senken. Der Personalrat beteiligte sich intensiv am Entscheidungsprozess und zog externe Berater zur Unterstützung hinzu. Seine Zielsetzung war, den Umfang des Personalabbaus zu reduzieren und betriebsbedingte Kündigungen zu verhindern. Tatsächlich wurde letzteres Ziel weitgehend erreicht (BruecknerBozetti/Schweizer 2000; Schütte 2004).

\subsection{DER WANDEL}

Die Phase der Sozialpartnerschaft innerhalb des LBK war jedoch nicht von Dauer. Als der LBK umstrukturiert wurde, befand sich die Landespolitik in einer Umbruch- phase. 2001 standen in Hamburg Wahlen an und Entscheidungsträger quer durch das politische Spektrum in Hamburg waren unzufrieden mit den Fortschritten, die der LBK hinsichtlich der Anpassung an die neuen Realitäten im Gesundheitswesen gemacht hatte. Das Top-Management argumentierte, die Krankenhäuser verursachten noch immer zu hohe Kosten und die Öffentlichkeit habe nun zwischen einer Einschränkung des Angebots und Kostensenkungen zu entscheiden (Schütte 2004). Zwei Kostenfaktoren waren bislang unberücksichtigt geblieben: die betriebliche Altersversorgung und überfällige Investitionen. Bei einem Verkauf rechnete der Senat mit Schwierigkeiten, einen guten Preis zu erzielen, da die städtischen Krankenhäuser nicht ohne Weiteres in gewinnbringende Unternehmen umzugestalten waren. Auf der anderen Seite würde ein wettbewerbsfähiges Gesundheitswesen der Stadt die Chance bieten, sich zu einer „Gesundheitsmetropole" zu entwickeln, die von den Synergieeffekten zwischen Kliniken, Geräteherstellern, der medizinischen Forschung und anderen im Gesundheitssektor tätigen Unternehmen profitieren würde (Lohmann 2004, S. 13-15). Nach den Wahlen von 2001 wurde die SPD als Regierungspartei abgewählt und an ihre Stelle trat eine Koalitionsregierung aus CDU, FDP und rechtsgerichteten Populisten (Schill-Partei). Die Ankündigung dieses Senats, 74,9\% der Anteile am LBK an ein Unternehmen der Privatwirtschaft veräußern zu wollen, wurde zum Ausgangspunkt einer neuen, lang andauernden Auseinandersetzung um den LBK. Das Vorhaben stieß auf den heftigen Widerstand von ver.di, dem Deutschen Gewerkschaftsbund (DGB) und dem Personalrat. Die Idee einer Privatisierung war nicht nur von der CDU getragen - Hamburgs oberste SPD-Führung reagierte 2004 mit dem Vorschlag, Geldmittel durch einen Börsengang des LBK zu generieren. Im Gegensatz zur damaligen SPD-Führung aber waren die Konservativen bereit, dieses Vorhaben zu Ende zu führen, ohne auch nur den Versuch einer Zusammenarbeit mit den Gewerkschaften zu unternehmen. Unterstützt von der SPD unterbreiteten die Gewerkschaften einen Gegenvorschlag mit dem Angebot, einen Partner für 49,9 \% der Anteile zu finden. Damit, so die Argumentation, würden die notwendigen Geldmittel beschafft und gleichzeitig bliebe die Kontrolle in der Hand der Stadt, die sicherstel- len würde, dass der gesellschaftliche Auftrag der LBK weitergeführt würde. Dagegen wandten die Konservativen ein, dass kein Investor bereit wäre, eine solche Regelung zu akzeptieren. Da Krankenhausbetreiber mit dem Kauf ein Risiko eingingen, so das Argument, seien sie auf Kontrolle angewiesen und müssten daher auch Mehrheitseigner sein. Ende des Jahres 2003 kam es zu einer entsprechenden Übernahmevereinbarung mit dem kommerziellen Klinikbetreiber Asklepios.

Die Gewerkschaftsseite erhob zahlreiche Einwände. Erstens befürchtete sie, dass die neuen Besitzer des LBK - so wie viele andere Krankenhäuser nach der Privatisierung - aus dem Flächentarifvertrag austreten und die wirksamen Mitbestimmungsstrukturen abschaffen würden; Asklepios eilte bereits der Ruf besonderer Gewerkschaftsfeindlichkeit voraus. Zweitens berge die Kontrolle eines kommerziellen Klinikbetreibers über etwa die Hälfte aller Krankenhausbetten in der Region die Gefahr von Pleiten und Schließungen anderer Krankenhäuser in der Region, da Asklepios sich auf die lukrativsten Patienten konzentrieren könnte. Drittens sei der Preis für die Anteile zu niedrig angesetzt. Schließlich kritisierten sie die mangelnde Transparenz des Verfahrens - es gab Zweifel daran, dass Asklepios tatsächlich das beste Angebot unterbreitet hatte - und dass die Regierung ein zu hohes Risiko einginge ( 75 Mio. $€$ des Kaufpreises waren an zu erzielende Erlöse geknüpft).

Der konfrontative Stil der Konfliktaustragung war nicht ideologisch motivierten Gewerkschaftern und Betriebsräten geschuldet. Sie waren sich mit der Regierung einig darüber, dass eine Qualitätssteigerung des LBK nicht ohne private Investitionen möglich sei, und sie hatten über Jahre ein Verhältnis vertrauensvoller Zusammenarbeit mit den Managern und dem SPD-geführten Senat gepflegt. Die neuen Gesundheits- und Haushaltsexperten im Senat hatten jedoch mit alternativen Konzepten zur Umstrukturierung oder gar mit Verhandlungen wenig im Sinn. Nach Diskussionen innerhalb von ver.di, Personalrat und DGB, kündigte ver.di-Landesbezirksleiter Wolfgang Rose eine neue Kampagne an, mit der er die Abgrenzung gegenüber der Politik des Senats demonstrierte. Dies sollte zu einem wichtigen identitätsstiftenden Moment für ver.di werden.

Mit einem Organisationsgrad von lediglich 10 bis $20 \%$ innerhalb des LBK und 
zudem ohne das Mittel des politischen Streiks befand sich die Gewerkschaft in keiner guten Ausgangslage für einen Arbeitskampf. Die Gewerkschaftsseite vermutete jedoch zu Recht, dass die Öffentlichkeit gegen eine Privatisierung war, und dass eine öffentliche Kampagne deshalb auf positive Resonanz stoßen würde. Trotz knapper Kassen wegen schwindender Mitgliederzahlen investierte die Gewerkschaft $360.000 €$ in eine Kampagne. Ver.di hielt die Kampagne für das geeignete Mittel, um trotz schwacher betrieblicher Verankerung $\mathrm{zu}$ einem öffentlichen Faktor zu werden, hierdurch das Prestige der Organisation zu stärken und Anreize zu Mitgliedschaft und gewerkschaftlichem, betrieblichem Engagement zu schaffen (Böhlke 2005).

\subsection{DIE KAMPAGNE "GESUNDHEIT IST KEINE WARE"}

Als Reaktion auf die neue Senatslinie ergriffen die Gewerkschaften unter dem Slogan „Gesundheit ist keine Ware!“ die Initiative zu einem der ersten auf ein breites Bündnis gestützten Volksentscheide in Hamburg. ${ }^{3}$ Neben den Beschäftigten stützte sich die Initiative auf einen breiten Zusammenschluss von Gewerkschaften, darunter auch der Marburger Bund, politischen Parteien sowie attac, der Bürgeraktion „Mehr Demokratie“, einzelnen Patienten sowie weiteren Mitstreitern. Die Bündnispartner hatten zum Teil sehr pragmatische Gründe für ihre Beteiligung. SPD und Grüne suchten Kontakt zu Kritikern des CDU-geführten Senats, „Mehr Demokratie“ ging es um das Anliegen, direktdemokratische Prozesse zu unterstützen, und ver.di hatte gemeinsame Interessen als Arbeitnehmervertretung mit dem Marburger Bund. Im Bündnis gab es auch Spannungen, wie z. B. die Ablehnung der Teilprivatisierung durch attac.

Anti-Privatisierungsaktivisten sammelten auf den öffentlichen Plätzen und in den Fußgängerzonen der Stadt mehr als 100.000 Unterschriften und organisierten zahlreiche Kundgebungen und Demonstrationen. Personalräte berichteten über einen enorm gewachsenen Zulauf zu Personalversammlungen in den Krankenhäusern. 12.000 der Unterschriften wurden allein in den Krankenhäusern gesammelt. Der Personalrat plante gegen den Widerstand des Managements, alle LBK-Angestellten zum ersten Mal zu einer Gesamtpersonalversammlung und einer Kundge- bung einzuladen, an der schätzungsweise eintausend Unterstützer und eintausend Beschäftigte teilnahmen. Nach einer langen Zeit des Co-Managements war dies ein wichtiger Schritt. Konservative Politiker reagierten und unterstellten im Gegenzug der Kampagne eine Art „VEB-Romantik“ (Hamburger Abendblatt 8. Mai 2003).

Gegen Ende des Jahres 2003 kollabierte die Schill-Partei und Neuwahlen für das Abgeordnetenhaus wurden anberaumt. Die Regierungskrise trat in dem Moment ein, als der Gesetzentwurf zur Privatisierung des LBK in die Bürgerschaft eingebracht wurde. Für die Aktivisten eröffneten sich dadurch ganz neue Perspektiven, denn eine im Eiltempo durchgeführte Abstimmung über die Gesetzesvorlage zur Privatisierung als letzte Amtshandlung der zusammengebrochenen Koalition war aus Sicht des Senats im anstehenden Wahlkampf nicht vermittelbar. Die anberaumten Bürgerschaftswahlen sollten am gleichen Tag stattfinden wie der Volksentscheid. Bei den Wahlen im Januar 2004 stimmten mehr als Dreiviertel der Wahlberechtigten für die Initiative der Gewerkschaften zum Stopp der Klinikprivatisierung. Parallel zu diesem großen Erfolg der Kampagne bescherten die Wähler allerdings der CDU einen Rekordsieg. Während der Stimmenanteil der SPD von 36,5 \% auf $30,5 \%$ zurückging, steigerte die CDU ihren Anteil von 26,2 \% auf 47,2 \%. Erstmals gab es in Hamburg eine absolute Mehrheit in der Bürgerschaft für die CDU.

Gestärkt durch diesen Wahlsieg ignorierte der neue CDU-Senat den Ausgang des Volksentscheides und lehnte ein Angebot von ver.di und des DGB ab, einen „Pakt der Vernunft" auszuhandeln. Das Hamburgische Verfassungsgericht stützte die Position des Senats, indem es entschied, der Volksentscheid sei zwar zunächst bindend, jedoch bleibe der Bürgerschaft die Möglichkeit, dieses Ergebnis wiederum durch ein neues Gesetz zu verändern und damit zu korrigieren. Kurz darauf brachte der Senat ein Gesetzesvorhaben auf den Weg, mit dem in Zukunft verhindert werden sollte, dass Abstimmungen über Volksentscheide zum gleichen Zeitpunkt stattfinden wie Kommunal- oder Bundestagswahlen. Zudem sollten die Unterschriftensammlungen ab sofort nur noch in amtlichen Räumen stattfinden - alles Regelungen, die höhere Hürden für die Verfahren der direkten Demokratie aufbauten. Im Sommer des Jahres 2005 ging der Senat noch einen
Schritt weiter und sprach im Pressegespräch über den Verkauf der verbliebenen Anteile an der LBK (taz 21.06.2005).

\subsection{DIE KAMPAGNE „RETTET DEN VOLKSENTSCHEID“"}

Die Gewerkschaft reagierte darauf mit einer zweiten Kampagne unter dem Motto „Rettet den Volksentscheid“ und hatte dabei die Unterstützung eines ähnlich zusammengesetzten Bündnisses wie bei der vorangegangenen Kampagne. Angeführt wurde diese nunmehr von einem pensionierten IG Metall-Funktionär, dem Vorstand des traditionsreichen bürgerlichen Vereins „Patriotische Gesellschaft von 1765 “ und dem Vorsitzenden der parteiunabhängigen Initiative zur Förderung der direkten Demokratie „Mehr Demokratie“. Rechtliche Beratung übernahm ein früherer Richter des Bundesverfassungsgerichtes. Die Aktivisten schlugen ein Bündel von Initiativen vor, um die Durchführung von Volksentscheiden zu vereinfachen und sie gesetzlich bindend zu machen. Dieser zweite Volksentscheid scheiterte am 14. Oktober 2007, trotz einer erneuten DreiviertelMehrheit der Abstimmenden, da die geforderte Wahlbeteiligung von $50 \%$ um ca. $10 \%$ verfehlt wurde. Nun wird parallel zur Bundestagswahl 2009 ein erneuter Versuch vorbereitet.

Nach dem Transfer der Krankenhäuser im Jahr 2005 bestätigten sich die Befürchtungen der Mitarbeiter von Asklepios: Der neue Arbeitgeber plante Entlassungen, verlangte längere Arbeitszeiten sowie niedrigeren Lohn und gründete darüber hinaus einen lokalen Arbeitgeberverband. Die Manager des Asklepios-Konzerns schlossen sich mit anderen, kleineren Krankenhäusern in der Region im Krankenhaus-Arbeitgeberverband Hamburg zusammen, dem im September 2005 auch der frühere LBK beitrat. Entgegen früherer Beteuerungen, die Arbeitsbedingungen an die im öffentlichen Dienst geltenden Vereinbarungen anzulehnen, hob das Management im Alleingang die wöchentliche Arbeitszeit für neue Mitarbeiter von 38,5 auf 42 Stunden

\footnotetext{
3 In Hamburg findet direkte Demokratie in einem mehrstufigen Verfahren statt. Zu Beginn steht die Volksinitiative, eine Bekundung öffentlichen Interesses durch Unterschriftensammlung. Daran schließt sich ein Volksbegehren an, das zu einem Volksentscheid, einer tatsächlichen Wahl führen kann.
} 
an und verlangte gleichzeitig die Kürzung des bezahlten Urlaubs, die Einstellung von mehr Zeitarbeitskräften und den Abbau von 1000 Arbeitsplätzen durch eine neuerliche Umstrukturierung der ausgelagerten Dienstleistungen.

\subsection{ERGEBNISSE DER KAMPAGNENARBEIT}

Im Oktober 2006 kam es nach zehnmonatigen Warnstreiks und harten Verhandlungen schließlich zum Kompromiss. Obwohl ver.di in der Kampagne gegen Privatisierung zunächst keine großen Mitgliederzuwächse erzielt hatte, hatte diese doch die Gewerkschaftsarbeit in den Kliniken entscheidend belebt. Die Kampagne schuf so die Voraussetzungen für die konfliktreiche Tarifauseinandersetzung, während der dann auch eine deutliche Stärkung der gewerkschaftlichen Mitgliederbasis erreicht wurde. Gegenüber dem Tarifvertrag des öffentlichen Dienstes (TVöD) fielen zwar einige Einmalzahlungen weg und der Anstieg leistungsbezogener Bonuszahlungen wurde verzögert, insgesamt wurde aber die Bindung an tarifliche Lohnsteigerungen und Arbeitszeitregelungen erreicht. Unerwähnt blieb im Tarifvertrag mit dem Krankenhausarbeitgeberverband Hamburg (TV$\mathrm{KAH}$ ) wie auch im TVöD der Verzicht auf betriebsbedingte Kündigungen, der Bestandteil aller früheren Vereinbarungen gewesen war.

Trotz dieser Zugeständnisse meldet ver.di, dass sich die Zahl der Gewerkschaftsmitglieder unter den LBK-Mitarbeitern parallel zu den Tarifverhandlungen verdoppelt habe und ferner ein Aktivitätsschub unter den Betriebsräten und Gewerkschaftsangehörigen am Arbeitsplatz festzustellen sei. Zugleich gingen die Auseinandersetzungen am Arbeitsplatz und in der politischen Arena weiter. Mehr als 1.500 frühere LBK-Mitarbeiter forderten bis Herbst 2007 ihr Rückkehrrecht in den öffentlichen Dienst der Stadt ein, das in der 1995 geschlossenen Vereinbarung festgeschrieben war. Darüber hinaus konnte Kritik an der Privatisierung nachhaltig in der Öffentlichkeit etabliert werden (vgl. z. B. Hamburger Abendblatt vom 21. Juni 2007).

Für Hamburgs Krankenhauspersonal und seine Vertreter lag das Problem nicht darin, dass eine konservative Regierung eine traditionsgemäß sozialdemokratische Regierung ersetzt hatte: beide politischen
Lager hatten sich für wettbewerbsorientierte Reformen im Gesundheitssektor und im öffentlichen Dienst stark gemacht. Die entscheidende Veränderung war vielmehr der Strategiewechsel weg von den eingespielten Pfaden der Sozialpartnerschaft hin zur Konfrontation mit der Gewerkschaft, den Senat und Arbeitgeber vollzogen. Damit wandelten sich auch Sinn und Zweck der Organe der Mitbestimmung: Während sie zuvor der Aushandlung von Vereinbarungen zwischen Stadt, Arbeitgeber und Gewerkschaft dienten, waren sie nunmehr Mittel der Informationsbeschaffung für Mitarbeitervertreter in einem Konfliktfall. Hatte die Gewerkschaft einst von einem guten Zugang zu hochrangigen Entscheidungsprozessen profitiert, so blieb sie jetzt davon ausgeschlossen.

Das Instrument der Volksabstimmung und der sie begleitenden Kampagne bot ver.di eine Handlungsalternative, nachdem die traditionellen Einflusskanäle zunehmend verschüttet waren. Der Wahlsieg der Konservativen ebnete zugleich den Weg für die Beteiligung von SPD und Grünen. Damit fanden sich nun auch Teile des Hamburger Establishments, die früher selbst städtische Betriebe privatisiert hatten, in einem außerparlamentarischen Bündnis wieder.

\section{Hamburg - kein Sonderfall}

Die in Hamburg von Arbeitgeberseite und Gewerkschaft eingeschlagenen Strategien sind im deutschen Gesundheitswesen kein Einzelfall. Die Umstrukturierung von Krankenhäusern ist ein allgemeiner Trend. Das Statistische Bundesamt meldet, dass eine wachsende Zahl öffentlicher Krankenhäuser entweder schließt oder privatisiert wird. Von 1991 bis 2005 ist die Zahl der kommerziell betriebenen Kliniken um 59 \% gestiegen, während die Zahl der Krankenhäuser in der öffentlichen Hand um 28 \% zurückgegangen ist. Die Gesamtzahl der Kliniken hat sich um $11 \%$ verringert. Während sich die Zahl der zu behandelnden Krankheitsfälle im gleichen Zeitraum um $16 \%$ erhöhte, verringerte sich die Mitarbeiterzahl um 3 \% (Statistisches Bundesamt 2007; Schulten 2006)

Der Wandel in der Verwaltungsstruktur beim LBK spiegelt nationale Trends zu unabhängigen Krankenhausmanagements und neuen Rechtsformen bei der Umstrukturierung von Krankenhäusern wider. So wurde etwa in Stuttgart das gesamte Krankenhausmanagement vorübergehend ausgelagert. Outsourcing von Dienstleistungsbereichen in Tochtergesellschaften ist ebenfalls eine weit verbreitete Strategie, die dem Management zahlreiche Vorteile bietet. Sie ermöglicht, Arbeitsplätze abzubauen, kapitalstarke und erfahrende Partner zu finden und von steuerlichen Vorteilen zu profitieren (Nagler et al. 2002, S. 120-154).

Die Privatisierung des Gesundheitswesens ist Teil umfassender Privatisierungen im öffentlichen Dienst und trägt zum weiteren Zerfall der traditionellen Tarif- und Arbeitsbeziehungen im öffentlichen Dienst bei (vgl. Dribbusch/Schulten 2007). Nicht nur in Hamburg besteht eine erhöhte Gefahr der Tarifflucht. Der Präsident der Deutschen Krankenhausgesellschaft hat wiederholt darauf hingewiesen, dass Krankenhäuser in Zukunft weniger Arbeitsplatzsicherheit bieten, längere Arbeitszeiten einführen und mehr Angehörige unterer Lohngruppen beschäftigen werden und dies entweder über Tarifreformen oder Tarifflucht durchzusetzen gedenken (z. B. Rocke 2003, 2004). Schließlich hat sich der Kostendruck mit der Einführung eines neuen Entgeltsystems nach Diagnosebezogenen Fallpauschalen (DRG) weiter verschärft. Danach erhält das Krankenhaus für die Behandlung einer bestimmten Erkrankung einen festgesetzten Betrag, unabhängig davon, welche Kosten dem Krankenhaus tatsächlich entstehen und wie lange die Therapie dauert. Davon profitieren Krankenhäuser, die betriebswirtschaftlich effizient arbeiten, weniger kosteneffizient arbeitende Kliniken sind dagegen von Insolvenz bedroht und werden von ihren Beschäftigten weitere Zugeständnisse verlangen (Schütte 2004). Insofern die marktorientierte Gesundheitspolitik engere Handlungsspielräume vor Ort schafft, kann man sowohl Konflikt als auch CoManagement auf betrieblicher Ebene, d.h. neue Muster von Verbetrieblichung in Deutschland, erwarten.

Die Antwort der Gewerkschaft auf die Privatisierung in Hamburg ist nicht ungewöhnlich. In der Tat gibt es kaum noch Krankenhausprivatisierungen, die nicht mit Protesten und zum Großteil auch mit direktdemokratischen Verfahren verhindert werden sollen. Auch in anderen kommunalen Krankenhäusern gehört die Kam- 
pagnenarbeit inzwischen zum taktischen Repertoire von ver.di. Bei einer Kampagne in Köln im Jahr 2003 - zu der auch öffentliche Kundgebungen gehörten - ging es um die Schaffung einer neuen Rechtsform für die halbautonome städtische Krankenhausgesellschaft durch die neu gewählte CDU-Regierung der Stadt. Nach Abschluss der Kampagne, einem Wechsel des Koalitionspartners von der FDP zu den Grünen und nach der Ernennung eines dem Arbeitnehmerflügel der CDU nahe stehenden Verwaltungsdirektors gelang es ver.di, eine Vereinbarung auszuhandeln, die die Mitbestimmung in der neu gebildeten Krankenhausgesellschaft stärkte. Im Herbst 2001 wendete sich in Stuttgart eine wesentlich schärfer geführte Kampagne mit Demonstrationen und mit Unterstützung der katholischen Betriebsseelsorger sowie anderer zivilgesellschaftlicher Organisationen zunächst gegen Entlassungen und eine Steigerung des Arbeitstempos in den Krankenhausküchen. Ab 2003 stand der Kampf gegen Pläne zur Schließung zweier Krankenhäuser (von ursprünglich dreien) und der Zentralisierung der Funktionsbereiche im Mittelpunkt der Kampagne. Als Resultat bewegten sich die Parteien Schritt für Schritt auf Verhandlungen zu, in denen Vereinbarungen über die Einzelheiten der Umstrukturierung zwischen Management und Personalräten getroffen werden sollten.

Kampagnenarbeit und bewegungsgewerkschaftliche Ansätze existieren nicht überall, wo Arbeitnehmervertreter nicht als Co-Manager fungieren. In Chemnitz etwa haben Arbeitnehmervertreter eine Art Sozialarbeitsfunktion übernommen, d. h. sie konzentrieren sich hauptsächlich auf die individuelle Beratung von betroffenen Arbeitnehmern, ohne dass eine intensive Mitgestaltung oder der Aufbau von Gegenmacht angestrebt wird. Die Geschäftsleitung hat, nachdem eine gemeinnützige Krankenhausgesellschaft (gGmbH) gegründet worden war, das Outsourcing forciert und Druck auf die Mitarbeiter ausgeübt, individuelle Arbeitsverträge mit einer untertariflichen Bezahlung zu unterzeichnen. Schließlich trat die Krankenhausgesellschaft im Jahr 2005 aus dem Arbeitgeberverband aus. Die Arbeitnehmervertreter konnten, obwohl im Stadtrat vertreten, nur in sehr geringem Maße Einfluss auf das Management nehmen. Die Ursachen für diese Schwäche sehen ver.diVertreter in der Rechtsform der gemein- nützigen Gesellschaft mit eingeschränkten Mitbestimmungsrechten, hoher Arbeitslosigkeit und einem von anti-gewerkschaftlichen Haltungen geprägten Umfeld.

\section{Bewegungsgewerkschaft- liche Ansătze in einer neoliberalen Welt}

Der Ansatz, Gewerkschaftsarbeit als soziale Bewegung zu interpretieren, ist eine Reaktion auf den immensen Druck, den auf Kosteneinsparung bedachte Arbeitgeber und neoliberale Regierungen auf die Gewerkschaften ausüben. Diese Strategie kann den Gewerkschaften neue Mitglieder zuführen und eine Erweiterung ihrer Machtbasis jenseits der Mitgliedschaft bewirken, zumindest auf lokaler Ebene. Kann damit aber auch der Trend zur Erosion gewerkschaftlicher Gestaltungsmacht umgekehrt werden, die das Modell Deutschland im Kern ausgemacht hat? Selbst wenn wir davon ausgehen, dass die Entwicklungen im Gesundheitssektor richtungweisend sind, kann die Antwort auf diese Frage nur dann ja lauten, wenn die neue Strategie sich auch auf nationaler bzw. transnationaler Ebene bewährt. Handlungsoptionen vor Ort sind zwar wichtig für die Entwicklung der Gewerkschaft als Organisation, aber sie bieten keine hinreichende Lösung der Probleme der Krankenhausbelegschaft, wenn die Ursachen kommunaler Liberalisierungspolitik in Brüssel und Berlin liegen.

Der neue Trend zur Kampagnenarbeit ist verbunden mit einem weitreichenden Wandel der Arbeitsbeziehungen im öffentlichen Dienst. Arbeitgeber im öffentlichen wie privaten Beschäftigungssektor ziehen sich im Zuge eines immer stärkeren Drangs nach Flexibilisierung und Kostenreduzierung aus den einstmals vorhandenen Strukturen der Zusammenarbeit und des Verhandelns zurück. Mit dem Zusammenbruch traditioneller Strukturen wird „insider unionism" (Turner/Cornfield 2007) ineffektiv oder sogar unmöglich, was in manchen Fällen dazu führt, dass Gewerkschaften sich als soziale Bewegung neu definieren. In diesem Artikel wurde beschrieben, wie eine einzelne lokale Gewerkschaftsgliederung als Reaktion auf die Herausforderungen neoliberaler Politik und angesichts sich auflösender Institutionen die Grundzüge einer sozialen Bewegung angenommen hat.

Ein Grund für den Zerfall der Institutionen in Deutschland ist der von politischen Parteien und Arbeitgebern ausgehende Druck, Reformen umzusetzen, die dem empfundenen Bedürfnis nach Wettbewerbsfähigkeit nachkommen. Das Vorgehen der Krankenhauskette Asklepios und des konservativen Hamburger Senats zeigt die Grenzen von Mitbestimmung als gewerkschaftliche Machtressource, da sie die Arbeitgeber nicht hindert, für ihre Personalpolitik wichtige Entscheidungen ohne Absprache zu fällen. Der Senat hatte sich unter der Annahme, die Gewerkschafter würden notwendige Maßnahmen blockieren, dazu entschieden, die Mitarbeitervertretung außen vor zu lassen. Der Senat lehnte das Modell der Sozialdemokraten $\mathrm{ab}$, die Daseinsvorsorge auf sozialpartnerschaftlichem Wege umzustrukturieren.

Ein weiterer Grund für den Strategiewechsel der Gewerkschaften lag in der Verfügbarkeit von Handlungsressourcen. Anstatt sich auf die üblichen Zielvorgaben und Vorgehensweisen zu berufen, die das System der institutionellen Arbeitsbeziehungen vorgibt, hat ver.di in Hamburg Neuland betreten und sich wesentlich kämpferischer gezeigt. Die örtlichen Vertreter von ver.di wandelten nicht so sehr auf vertrauten Pfaden und bauten weniger auf eine erprobte Machtbasis. Sie machten sich auf eine ergebnisoffene Suche nach neuen Ansatzpunkten für ihre Einflussnahme. Das heißt, sie suchten nach kontroversen Themen, die bei weit über die Mitgliedschaft der Gewerkschaft hinausgehenden Teilen der Öffentlichkeit auf Interesse stoßen, und sie bauten neue Netzwerke auf. Zwei wesentliche Ziele der Kampagne lagen jenseits der unmittelbaren Bedürfnisse der Krankenhausbeschäftigten nach Arbeitsplatzsicherheit und guten Arbeitsbedingungen: es ging um direkte Demokratie auf lokaler Ebene und die staatliche Verantwortung für die Daseinsvorsorge. Die Bereitschaft und Fähigkeit der Arbeitnehmervertreter in anderen Städten, eine solche Strategie umzusetzen, ist in hohem Maße von politischen und wirtschaftlichen Faktoren vor Ort konditioniert.

Auch wenn das Hamburger Beispiel begründeten Anlass für Optimismus gibt, sollte es nicht überbewertet werden. Bewegungsgewerkschaftliche Ansätze sind in Deutschland bisher noch auf lokale Einzelfälle beschränkt und auch bei ver.di kein in- 
tegraler Teil einer übergeordneten Strategie zur gewerkschaftlichen Neuorganisierung. Örtliche Auseinandersetzungen über Tarifverträge und Privatisierungen finden getrennt von Debatten über Finanz-, Steuerund Wettbewerbspolitik statt, die nationa- le, europäische oder gar globale gewerkschaftliche Strukturen einbeziehen. Ohne eine solche Einbindung und eine Koordination gewerkschaftlicher Erneuerung auf nationaler oder gar internationaler Ebene ist zwar eine Revitalisierung einzelner
ver.di-Bezirke entlang von bewegungsgewerkschaftlichen Konzepten denkbar. Auf sich alleine gestellt, werden lokale Gewerkschaftsorganisationen aber nicht in der Lage sein, dem allgegenwärtigen neoliberalen Kurs wirksam entgegenzutreten.

\section{LITERATUR}

Baccaro, L./Hamann, K./Turner., L. (2003): The Politics of Labour Movement Revitalization: The Need for a Revitalized Perspective, in: European Journal of Industrial Relations 9, S.119-133

Bispinck, R. (2006): Mehr als ein Streik um 18 Minuten - Die Tarifauseinandersetzungen im öffentlichen Dienst 2006, in: WSI-Mitteilungen 7, S. 374-381

Böhlke, N. (2005): Direktdemokratische Verfahren als strategisches Instrument der Gewerkschaften zur Verhinderung von Privatisierung. Diplomarbeit, Universität von Hamburg

Brandt, T./Drews, K./Schulten, T. (2007): Liberalisierung des deutschen Postsektors. Auswirkungen auf Beschäftigung und Tarifpolitik, in: WSIMitteilungen 5, S. 266-273

Brueckner-Bozetti, P./Schweizer, S. (Hrsg.) (2000): Krankenhausmanagement und Arbeitnehmerstrategie: ein Projekt der Gewerkschaft ÖTV und des Gesamtpersonalrates des LBK Hamburg zur Unternehmens- und Beschäftigungssicherung, Frankfurt am Main

Doellgast, V./Greer, I. (2007): Vertical Disintegration and the Disorganization of German Industrial Relations, in: British Journal of Industrial Relations 45, S. 55-76

Dörre, K./Röttger, B. (2006): Im Schatten der Globalisierung. Strukturpolitik, Netzwerke und Gewerkschaften in altindustriellen Regionen, Wiesbaden

Dribbusch H./ Schulten, Th. (2007): The End of an Era: Structural Changes in German Public Sector Collective Bargaining, in: Leisink, P. et al. (Hrsg.): Industrial Relations in the New Europe: Enlargement, Integration and Reform, Cheltenham, S. 155-176

Huhn, J. (2001): Die Schlecker-Kampagne 1994-1995: Gewerkschaft als soziale Bewegung, Mannheim

Johnston P. (1994): Success while others fail, Ithaca

Katz H./ Darbishire, O. (2000): Converging Divergences, Ithaca

Lash, S./Urry, J. (1988): The End of Organized Capitalism, Gerards Cross Lohmann, H. (2004): Das Kapitalproblem des LBK muss gelöst werden, in: in f\&w - führen und wirtschaften im Krankenhaus 21, S. 13-15
Lopez, S. (2004): Reorganizing the Rust Belt: an inside study of the American labor movement, Berkeley

Moody K. (1997): Workers in a Lean World, London

Nagler, B./Haslinger, S./Meurer, P. (2002): Mitbestimmungsvereinbarungen in öffentlichen Unternehmen mit privater Rechtsform, Baden-Baden Rocke, B. (2003): Flucht aus dem BAT und aus der Zusatzversorgung?, in: das Krankenhaus 95, S. 449-453

Rocke, B. (2004): Was wurde bei den Verhandlungen um BAT bisher erreicht?, in: das Krankenhaus 96, S. 875-878

Seifert, H./Massa-Wirth, H. (2005): Pacts for employment and competitiveness in Germany, in: Industrial Relations Journal, 3, S. 217-240

Schulten, T. (2006): Country reports on liberalisation and privatisation processes and forms of regulation. Liberalisation, privatisation and regulation in the German healthcare sector/hospitals, Deliverable 1 for the Project Privatisation of Public Services and the Impact on Quality, Employment and Productivity (PIQUE), Düsseldorf

Schütte, G. (Hrsg) (2004): Heinz Lohmann. Mut zum Wandel: Texte zur Entwicklung der Gesundheitswirtschaft, Melsungen

Statistisches Bundesamt (2007): Gesundheitswesen: Grunddaten der Krankenhäuser, Bonn

Statistikamt Nord (2006): 500 Jahreszeitreihen. Erwerbstätigkeit. Erwerbstätige Hamburger und Hamburgerinnen nach Sozialstatus und Wirtschaftsbereichen, Hamburg

Streeck, W. (2001): High Equality, Low Activity, in: Industrial and Labor Relations Review 3, S. 698-706

Thelen, K./Kume, I. (2006): Coordination as a political problem in coordinated market economies, Governance 1, S. 11-42

Turner, L. (1991): Democracy at Work, Ithaca

Turner, L./Cornfield, D. (Hrsg.) (2007): Labor in the New Urban Battlegrounds: Local Solidarity in a Global Economy, Ithaca

Voss, K./Sherman, R. (2000): Breaking the Iron Law of Oligarchy: Union Revitalization in the American Labor Movement, in: American Journal of Sociology 2, S. 303-349 